\title{
STUDY OF THYROID PROFILE IN GERIATRIC TYPE-2 DIABETICS IN JHARKHAND
}

\author{
PANKAJ KUMAR JHA ${ }^{1 *}$, GUNJAN KUMAR MANDAL ${ }^{2}$
}

${ }^{1}$ Department of Biochemistry, Rajendra Institute of Medical Sciences, Bariatu, Ranchi - 834 009, Jharkhand, India. ${ }^{2}$ Department of Biochemistry, Prasad Institute of Medical Sciences, Banthara, Lucknow - 226 401, Uttar Pradesh, India. Email: sanjujh35@gmail.com

Received: 09 January 2017, Revised and Accepted: 24 January 2017

ABSTRACT

Objective: To study the association of the two endocrinal disorders (diabetes mellitus and thyroid disease) in randomly selected geriatric patients.

Methods: The study includes 80 patients in geriatric age groups with Type-2 diabetes mellitus visiting the medicine outpatients department. Thyroid profile was assayed in Type-2 diabetic patients.

Results: It was noted that thyroid dysfunction was prevalent in 41 (51.25\%) out of the 80 geriatric age group patients with 31 (38.75\%) being hypothyroidism, 7 (8.7\%) having subclinical hypothyroidism, and 3 (3.7\%) having a hyperthyroid state.

Conclusion: The study shows that screening should be strongly recommended for all Type-2 diabetics to rule out thyroid dysfunction in patients with increasing age.

Keywords: Thyroid profile, Geriatric patients, Diabetes mellitus.

(C) 2017 The Authors. Published by Innovare Academic Sciences Pvt Ltd. This is an open access article under the CC BY license (http://creativecommons. org/licenses/by/4. 0/) DOI: http://dx.doi.org/10.22159/ajpcr.2017.v10i4.17000

\section{INTRODUCTION}

Thyroid disease (TD) is common in the general population and its prevalence increases with age [1]. Altered serum thyroid-stimulating hormones (TSHs) levels with normal or altered thyroid hormone, free triiodothyronine (FT3) and free thyroxine (FT4) is defined as thyroid dysfunction [2]. Different TDs include Hashimoto's thyroiditis, hyperthyroidism, and hypothyroidism [3]. Hypothyroidism is a state in which the thyroid organ does not make enough thyroid hormone. Iodine insufficiency is frequently referred to as the most well-known reason for hypothyroidism overall, however, it can be brought about by numerous different variables. It can come about because of the absence of a thyroid organ or from iodine-131 treatment, and can likewise be connected with expanded anxiety [4]. The assessment of thyroid function by modern assays is both reliable and inexpensive. Screening for thyroid dysfunction is indicated in certain high-risk groups such as the elderly and those with other endocrinal disorders especially diabetes mellitus [1]

Diabetic patients have a higher prevalence of thyroid disorders compared with the normal population. A number of reports have also indicated a higher than normal prevalence of thyroid disorders in Type-2 diabetic patients with hypothyroidism being the most common thyroid disorder and especially in older women [1].

Hypothyroidism is usually autoimmune in origin presenting as either primary atrophic hypothyroidism or Hashimoto's thyroiditis. Thyroid failure secondary to radioactive iodine therapy or thyroid surgery is also common. Rarely, pituitary or hypothalamic disorders can result in secondary hypothyroidism [1].

Millions of people in India are hypothyroid and are on hormone replacement therapy. By contrast, hyperthyroidism is much less common with a female to male ratio of 9:1. Grave's disease is the most common cause and primarily affects young adults. Toxic multi-nodular goiter tends to affect the older age groups. Moreover, nodular thyroid structure and parenchymatous goiter occur more frequently in Type- 2 diabetics [5].
According to the WHO and UN, 2001, people aged 45-59 years are considered "middle aged" and those above 60 years are considered to be geriatrics, though there is no fixed or mandatory criteria or guideline. In the real world, especially, where jobs and pension schemes and other such scenarios where benefits are concerned, people over 65 years are considered to be geriatric [6]. In this study, though people over 60 years will be considered as geriatric.

The presence of thyroid dysfunction may affect glycemic control in diabetics. Hyperthyroidism is typically associated with worsening glycemic control and increased insulin requirements. There is underlying increased hepatic gluconeogenesis, rapid gastrointestinal absorption of glucose, and probably increased insulin resistance also. Indeed, thyrotoxicosis may unmask latent diabetes [1].

Although wide ranging changes in carbohydrate metabolism are seen in hypothyroidism, clinical manifestations of these abnormalities are seldom conspicuous. However, the reduced rate of insulin degradation may lower the exogenous insulin requirements. The occurrence of hypoglycemic states is uncommon in isolated thyroid hormone deficiency and should raise the doubt of hypopituitarism in a patient with hypothyroidism. More importantly, hypothyroidism is accompanied by a variety of abnormalities in lipid metabolism including elevated triglyceride and low-density lipoprotein (LDL) concentrations. Even subclinical hypothyroidism can worsen the coexisting dyslipidemia commonly found in Type- 2 diabetics and hence further increase the risks of cardiovascular events. Adequate hormone replacement will reverse the lipid abnormalities $[1,5]$.

All steps of lipids metabolism are involved by thyroid hormones leading to development of qualitative and quantitative changes of serum lipids [7].

In conclusion, thyroid dysfunction is common in diabetics and can produce significant metabolic disturbances with serious outcomes. Therefore, regular screening for thyroid abnormalities in all diabetic patients will allow early detection and effective treatment of thyroid dysfunction to prevent morbidity. A sensitive serum TSH assay is 
Table 1: Thyroid status of the patients studied

\begin{tabular}{lllll}
\hline Age group & Normal euthyroid & Hypothyroidism & Subclinical hypothyroidism & Hyperthyroidism \\
\hline Geriatric $(>60$ year $) \mathrm{n}=80$ & $39(48.75 \%)$ & $31(38.75 \%)$ & $7(8.7 \%)$ & $3(3.7 \%)$ \\
\hline
\end{tabular}

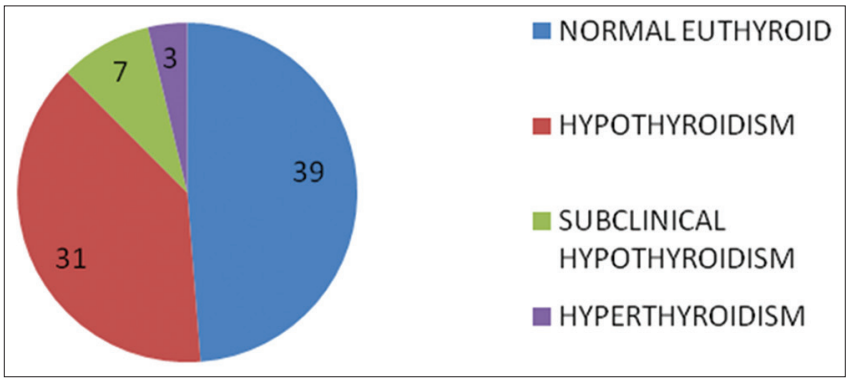

Fig. 1: Thyroid dysfunction in geriatric Type-2 diabetic patients

the screening test of choice. In Type- 2 diabetic patients, a TSH assay should be done at diagnosis and then repeated at least every 5 years (if euthyroid) [1].

\section{METHODS}

The study was performed after approval from the Institutional Ethical Committee and written consent was obtained from all participant in our study.

\section{Patients selection}

This study conducted between May 2014 and November 2015 in 80 previously diagnosed Type- 2 diabetic patients attending the outpatient department and admitted in-patients of Medicine Department of RIMS, Ranchi, were randomly selected and screened for thyroid dysfunction after obtaining a detailed history, also pertaining to thyroid and diabetes-related symptoms, and then subjecting them to an equally detailed general physical examination.

\section{Inclusion criteria}

A total of 80 patients in the geriatric age group with Type-2 diabetes mellitus.

\section{Exclusion criteria}

1. Patients with Type- 1 diabetes mellitus

2. Patients on hormone replacement therapy

3. Patients on steroids

4. Pregnant women

5. Patients on drugs known to cause hypothyroidism (propranolol, iopanoic acid, iodide, amiodarone, salicylates, phenytoin, glucocorticoids, lithium, amphetamines, sertraline, aminoglutethimide, dopamine, somatostatin, octreotide, interleukins, heroin)

6. Patients with cirrhosis of liver

7. Patients with renovascular hypertension or renal failure

8. Patients with heart failure.

\section{Sample collection}

Fasting blood samples were collected for estimation of blood glucose and thyroid profile. Blood glucose was done by hexokinase method and thyroid profile by chemiluminescent microparticle immunoassay method.

\section{Statistical analysis}

All data were expressed as the mean and standard deviation, the SPSS 20 software was used for the statistical analysis. The statistical significance was analyzed in the subjects using the Student's t-test. Significance was considered for all tests $(\mathrm{p}<0.05)$. For Student's t-test significance represented as $\left({ }^{*}\right)$ less significant $(\mathrm{p}<0.05),\left({ }^{* *}\right)$ significant $(\mathrm{p}<0.01)$, and $(* * *)$ highly significant $(\mathrm{p}<0.001)$.

\section{RESULTS}

It was noted that thyroid dysfunction was prevalent in 41 (51.25\%) in the studied patients with 31 (38.75\%) being hypothyroid, 7 (8.7\%) having subclinical hypothyroidism, and $3(3.7 \%)$ having a hyperthyroid state (Table 1 and Fig. 1).

Thyroid dysfunction was predominantly seen in females (77 in the studied age groups) with the prevalence rising with increasing age.

Maximum number of patients, 37 (46.25\%) had a 10-14 years duration of diabetes.

A total of 38 patients out of 80 in the study group had dyslipidemia of which $21(55.26 \%)$ patients were found to have significantly altered serum lipids levels. Only 3 (7.8\%) patients out of 38 with dyslipidemia also had subclinical hypothyroidism.

The prevalence of anteroseptal wall ischemia in the studied age group was observed to be $7(17.0 \%)$. Lateral wall ischemia was seen to be present in $6(14.6 \%)$ patients. Inferior wall ischemia was revealed in the electrocardiograms of $11(26.8 \%)$ patients.

\section{DISCUSSION}

Thyroid dysfunction was predominantly seen in females (a total of 80 patients with 77 in the study groups) with the prevalence rising with increasing age.

A significant association was seen between increasing body mass index (BMI) values with the prevalence of TD where 26 (78\%) of the 32 hypothyroid patients in the study group had a BMI $>25$ (Grade I and II obesity) with diabetes.

Both diabetes and TD as separate entities lead to significant cardiovascular morbidity due to alterations in the metabolism of lipids leading to increased atherogenicity. The presence of both endocrinal disorders in patients heightens the risk of morbidity and also mortality manifold which is evident by the presence of dyslipidemia in most of these patients and the direct effect of both in causing ischemic heart disease. 38 (47.5\%) patients in study groups were found to have altered serum lipid values of which 19 (23.7\%) patients had hypothyroidism.

Thyroid function tests done in a study population of 298 Type-2 diabetics conducted by Nobre et al. in 2002, in Europe showed that $38(12.7 \%)$ of them suffered from thyroid dysfunction- $10.7 \%$ had hypothyroidism $\left(>2 / 3^{\text {rd }}\right.$ with subclinical hypothyroidism $)$ and $2 \%$ had hyperthyroidism. In 31 cases (10.4\%), the diagnosis was performed "de nova." TD was more prevalent among females and the elderly [8].

In a study conducted by Radaideh et al. with 908 Type- 2 diabetics and 304 non-diabetics at Amman, Jordan, the overall prevalence of TD in diabetics was found to be $12.5 \%$ of which $5.9 \%$ were known to have TD and the rest (6.6\%) were newly diagnosed cases as a direct result of screening. The most common was subclinical hypothyroidism (4.1\%). The prevalence of TD was $6.6 \%$ in the control group [9].

An Indian study done at GND Hospital, Amritsar by Bal et al. in 2003, with 184 Type-2 diabetics showed that TD was present in 78 (40.4\%) cases (50 males and 28 females) but autoimmune thyroiditis (AT) was present in $32(17.4 \%)$ cases (8 males and 24 females). There was a positive correlation with the age of the patients in the TD group, but there was no significant correlation found with complications of 
diabetes. There was no correlation of age, severity or complications in the AT group but these findings were significantly more in females [10].

In a study sample of 100 Type- 2 diabetics at Chennai, the prevalence of TD was found to be $15 \%$ with subclinical hypothyroidism - $11 \%$, hypothyroidism - 1\%, subclinical hyperthyroidism - $2 \%$ and hyperthyroidism - 1\%. The prevalence was higher than that in the general population and in females [11].

In another study of 120 Type- 2 diabetics conducted at Hyderabad by Ramaswamy in 2003, hypothyroidism was seen in 32 (27\%) cases of which $10 \%$ had subclinical hypothyroidism with $80 \%$ of them being females. $70 \%$ of the patients with hypothyroidism were between 40 and 60 years of age. Only in $1 \%$ cases, the appearance of hypothyroidism had preceded Type- 2 diabetes and in $2 \%$ hypothyroidism cases the titers of antimitochondrial antibody were significant and goiter was noted in $2 \%$ of the patients [12]

The Colorado TD Prevalence Study conducted under Canaris in the year 2000 showed that the consequences of untreated subclinical hypothyroidism include cardiac dysfunction or adverse cardiac endpoints (atherosclerotic disease and cardiovascular mortality), elevation of total and LDL cholesterol, systemic hypothyroid symptoms or neuropsychiatric symptoms, progression to overt symptomatic hypothyroidism [13].

Zondek [14] detected an increased association of coronary heart disease in hypothyroidism. In a study by Sawin et al. [15] on elderly women, 3\% were found to be hypothyroid. In another study conducted by Bahemuka and Hodkinson [16], 2.3\% of elderly women had hypothyroidism. Kutty et al. [17] showed that clinically overt hypothyroidism in patients is associated with hyperlipidemia

\section{CONCLUSION}

Thyroid dysfunction is seen in a significant percentage of the previously diagnosed Type- 2 diabetics with the most predominant dysfunction being overt hypothyroidism followed by subclinical hypothyroidism, females being predominantly affected. The prevalence of hyperthyroidism was very less in these patients. Hypothyroidism was seen more in patients with increasing age was noticed with a higher prevalence among the older patients in the geriatric age mark and was associated with an increase in BMI.

The presence of secondary dyslipidemia due to hypothyroidism and increased BMI as a consequence of previously undiagnosed hypothyroidism is a major risk factor for atherosclerosis and its accompanying vascular complications especially if these patients are affected with a dual endocrinological disorder, the other being diabetes mellitus.
In conclusion screening should be strongly recommended for all diabetes to rule out thyroid disfunction especially in patients with increasing age and obesity to timely diagnose and prevent major cardiovascular morbidity and mortality.

\section{REFERENCES}

1. Wu P. Thyroid disease and diabetes. Clin Diabetes 2000;18(1):1-10.

2. Gyawali P, Takanche JS, Shrestha RK, Bhattarai P, Khanal K, Risal P, et al. Pattern of thyroid dysfunction in patients with metabolic syndrome and its relationship with components of metabolic syndrome. Diabetes Metab J 2015;39(1):66-73.

3. Sugumar S, Aparajita. Genome-wide analysis of long non-coding RNA (LNCRNA) of autoimmune thyroid diseases using bioinformatics approaches. Int J Pharm Pharm Sci 2016;8(6):142-50.

4. Azharuddin M, Atif M, Ahmed M, Bakhtiary S, Ibrahim M. Evaluation of anti-thyroid activity of ficus racemosa Linn bark in male rats. Int $\mathrm{J}$ Pharm Pharm Sci 2015;7(3):118-22.

5. Sawin CT, Chopra D, Azizi F, Mannix JE, Bacharach P. The aging thyroid. Increased prevalence of elevated serum thyrotropin levels in the elderly. JAMA 1979;242(3):247-50.

6. WHO Definition of an Older or Elderly Person. WHO Health Statistics and Health Information System. Available from: http://www.who.int/ healthinfo/survey/ageingdefnolder/en/index.html.

7. Shashi A, Devi U, Singla S. Association between hypothyroidism and metabolic syndrome in Type 2 diabetic patients: A cross sectional study: Int J Basic Appl Med Sci 2013;3(1):98-109.

8. Nobre EL, Jorge Z, Prastas S, Silva C, Castro JJ. Profile of the thyroid function in a population with Type 2 diabetes. Endocr Abstr 2002;3:298.

9. Radaideh AR, Nusier MK, Amari FL, Bateiha AE, El-Khateeb MS, Naser AS, et al. Thyroid dysfunction in patients with Type 2 diabetes mellitus in Jordan. Saudi Med J 2004;25(8):1046-50.

10. Bal BS, Kaur PP, Singh BM, Gurpal S, Singh SP. Prospective analysis of thyroid abnormalities in cases of Type 2 diabetes mellitus. J Assoc Physicians India 2003;51:1165-6.

11. Lotz H, Salabè GB. Lipoprotein(a) increase associated with thyroid autoimmunity. Eur J Endocrinol 1997;136(1):87-91.

12. Aviram M, Luboshitzky R, Brook JG. Lipid and lipoprotein pattern in thyroid dysfunction and the effect of therapy. Clin Biochem 1982;15(1):62-6

13. Ayala AR, Danese MD, Ladenson PW. When to treat mild hypothyroidism. Endocrinol Metab Clin North Am 2000;29(2):399-415.

14. Zondek H. Association of myxedema heart and arteriosclerotic heart disease. J Am Med Assoc 1959;170(6):1920-1.

15. Sawin CT, Geller A, Kaplan MM, Bacharach P, Wilson PW, Hershman JM. Low serum thyrotropin (thyroid-stimulating hormone) in older persons without hyperthyroidism. Arch Intern Med 1991;151(1):165-8.

16. Bahemuka M, Hodkinson HM. Screening for hypothyroidism in elderly inpatients. Br Med J 1975;2(5971):601-3.

17. Kutty KM, Bryant DG, Farid NR. Serum lipids in hypothyroidism - A re-evaluation. J Clin Endocrinol Metab 1978;46(1):55-6. 